\title{
Microbial Population and Soil Health under Organic Cotton Production System
}

\author{
Rudragouda F. Channagouda*
}

Krishi Vigyan Kendra, Babbur Farm, Hiriyur University of Agricultural and Horticultural Sciences, Shivamoga, Karnataka, India

*Corresponding author

\section{A B S T R A C T}

\section{Keywords}

Yield, Fibre length,

Energy use

efficiency,

Available NPK,

PSM, N-fixers

Article Info

Accepted:

26 February 2019

Available Online:

10 March 2019
A field experiment was carried out at MARS, University of Agricultural Sciences, Dharwad during Kharif, 2010 -11 and 2011-12 to study the "Nutrient management options for sustainable organic cotton production" The results of the two years pooled data revealed integrated application of EC (1/3) + VC (1/3) + gliricidia GLM (1/3) equivalent to RDF with foliar spray of panchagavya @ 5\% recorded significantly available soil N, $\mathrm{P}_{2} \mathrm{O}_{5}$ and $\mathrm{K}_{2} \mathrm{O}\left(284,29.7,330 \mathrm{~kg} \mathrm{ha}^{-1}\right.$, respectively), soil organic carbon $\left(5.7 \mathrm{~g} \mathrm{~kg}^{-1}\right.$, respectively), bacteria $\left(71.63 \mathrm{cfuX} 10^{6} / \mathrm{g}\right.$ of soil), fungi $\left(23.71 \mathrm{cfuX} 10^{3} / \mathrm{g}\right.$ of soil), actinomycetes $\left(37.32 \mathrm{cfuX} 10^{2} / \mathrm{g}\right.$ of soil), $\mathrm{N}_{2}$-fixers $\left(34.55 \mathrm{cfuX} 10^{3} / \mathrm{g}\right.$ of soil), P-solubilizer (27.1 $\mathrm{cfuX} 10^{3} / \mathrm{g}$ of soil), phosphatase $(24.52 \mu \mathrm{g} \mathrm{pnpTPF} / \mathrm{g}$ of soil/hr) and dehydrogenase enzyme activity (10.88 $\mu \mathrm{g} \mathrm{TPF} / \mathrm{g}$ of soil/day) and soil respiration rate $(10.95 \mathrm{mg}$ of $\mathrm{CO}_{2} / \mathrm{hr} / 100 \mathrm{~g}$ soil) over FYM @ $5 \mathrm{t} \mathrm{ha}^{-1}+\mathrm{RDF}$.

\section{Introduction}

The continuous use of chemical fertilizers for attaining main goal of maximum yield but not considered sustainable yield in long term and restless monocropping deplete the inherent fertility of the soil much faster than it can be replenished and reduce yield. Exclusive use of chemical fertilizers and pesticides in agriculture not only shattered the hope of farmers, but also received sever criticism from environmentally conscious people who opined that increase in agricultural production achieved at the cost of soil health. To the maximum extent organic production rely on crop green manures, organic manures, biofertilizers and other nutrient sources like use of fermented organic nutrients mainly panchagavya, jeevamruth, cow urine, vermiwash, bio-digester etc, are being popular among the farmers for sustainable crop production and maintain soil health on sustainable basis.

In India, cotton is grown over an area of about $11.25 \mathrm{~m}$.ha with a total production of $34.23 \mathrm{~m}$. bales (Anon., 2016). India ranks fifth in area and third in production of cotton after USA 
and China. The productivity of cotton is 510 $\mathrm{kg}$ of lint $\mathrm{ha}^{-1}$ which is much lower than the world average of $621 \mathrm{~kg} \mathrm{ha}^{-1}$. World organic cotton production is 241276 MT (1.1 million bales) grown on 0.46 million ha of land. The Organic Cotton Farm and Fiber Report reveals that India, Syria, and Turkey are the leading organic cotton producers in the world. India remains the top producer of organic cotton, out of the twenty-three organic cottonproducing cowntries, growing $80 \%$ of the fiber grown worldwide. In India, organic cotton is grown over an area of about 57,705 ha with a production of $2,58,823$ bales which is $25 \%$ of world share. The global retail market of organic cotton has increased from 583 million to 4.3 billion in 2009 with an annual growth rate of $3.5 \%$ (Anon., 2014). The information on nutrient management practices through organics for sustainable organic cotton production is very much limited which is a need of the hour. The proposed study aims at developing integrated organic nutrient management practices which is one of the important components to sustain the cotton production and soil productivity in terms of fertility and beneficial microorganism load.

\section{Materials and Methods}

A Field experiment was conducted at MARS, Dharwad during 2010 and 2011 to study the "Nutrient management practices for organic cotton production". The soil of the experiment site was clay, having medium carbon $(0.41 \%)$ and available NPK (264.70:24.80:285.30 NPK $\mathrm{kg} \mathrm{ha}^{-1}$ ). The experiment was laid out in split plot design with three replication. The main plot comprises of five manurial treatments as M1: Recommended dose of fertilizer $(\mathrm{RDF})\left(80: 40: 40 \mathrm{~N}: \mathrm{P}_{2} \mathrm{O}_{5}: \mathrm{K}_{2} \mathrm{O} \mathrm{kg} \mathrm{ha}{ }^{-1}+\mathrm{FYM}\right.$ @5 $\mathrm{t} \mathrm{ha}^{-1}$ ), M2: Crop residues equivalent to $50 \%$ RDN with compost culture + vermicompost equivalent to $50 \%$ RDN M3: Crop residues equivalent to $50 \%$ RDF with
Compost culture + vermicompost equivalent to $50 \%$ RDF, M4: Compost equivalent to $50 \%$ $\mathrm{RDN}+$ vermicompost equivalent to $50 \%$ RDN, M5: Compost equivalent to 50\% RDF + vermicompost equivalent to $50 \% \mathrm{RDF}$ and sub plot consists of six green manures treatments are S1: Gliricidia GLM mulch @ $7.5 \mathrm{t} \mathrm{ha}^{-1,} \mathrm{~S} 2$ : Gliricidia GLM mulch @ 7.5 t ha $^{-1}+$ Soil application of jeevamrutha@500 lit ha ${ }^{-1}$ at sowing, 30, 60 and 90 DAS, S3: Lucerne GM alone as inter crop (1:2 row proportion), S4: Lucerne GM as inter crop + Soil application of Jeevamrutha@500 lit ha ${ }^{-1}$, S5: Sunnhemp GM alone as inter crop (1:2 row proportion), S6: Sunnhemp GM as inter crop + Soil application of jeevamrutha @ 500 lit ha ${ }^{-1}$, two control treatments are T1: Recommended dose of fertilizer (RDF)(80:40:40 N: $\mathrm{P}_{2} \mathrm{O}_{5}: \mathrm{K}_{2} \mathrm{O} \mathrm{kg}$ $\mathrm{ha}^{-1}+$ FYM @ $5 \mathrm{t} \mathrm{ha}^{-1}$ ) and T2: Recommended dose of fertilizer (RDF)(80:40:40 N: $\mathrm{P}_{2} \mathrm{O}_{5}: \mathrm{K}_{2} \mathrm{O}$ $\mathrm{kg} \mathrm{ha}^{-1}$ ) only. The seeds were treated with cow urine, Azospirilum, Phosphate solubalizing bacteria, Pseudomonas striata, Trichoderma and cow dung slurry before sowing. The seeds were hand dibbled with two cotton seeds per hill on 12, july, 2010 and 15 june, 2011. Nitrogen estimation was done by Kjeldahl's method, phosphorus by vanado molybdate phosphoric yellow colour method and potassium by flame photometric method. The soil adhering to the roots was carefully collected and used for enumeration of actinomycetes, by Kusters agar for actinomycetes. The microbial populations were expressed as number of colony farming units per gram dry weight of soil.

\section{Results and Discussion}

The uptake of major nutrients mainly nitrogen, phosphorus and potassium differed significantly due to organic manures. Among the organic manurial treatments, application of EC $(1 / 3)+$ VC $(1 / 3)+$ gliricidia GLM (1/3) equivalent to RDF recorded significantly higher uptake of $\mathrm{N}, \mathrm{P}_{2} \mathrm{O}_{5}$ and $\mathrm{K}_{2} \mathrm{O}$ (72.53, 
14.26 and $77.3 \mathrm{~kg} \mathrm{ha}^{-1}$, respectively) over EC $(1 / 3)+\mathrm{VC}(1 / 3)+$ GLM (1/3) equivalent to RDN. Among the recommended nutrient practices, integrated application of FYM @ $5 \mathrm{t}$ $\mathrm{ha}^{-1}+\mathrm{RDF}$ recorded significantly higher uptake of $\mathrm{N}, \mathrm{P}$ and $\mathrm{K}(79.0,17.1$ and $80.9 \mathrm{~kg}$ $\mathrm{ha}^{-1}$, respectively) accounting for $16.52,36.77$ and 5.35 per cent, respectively (Table 2) higher over EC (1/3) + VC (1/3) + GLM (1/3) equivalent to RDN. The increase in total dry matter production and seed cotton yield could be ascribed to increased uptake of nutrients (Lokesh et al., 2008). Foliar spray of panchagavya @5\% recorded significantly higher uptake of N, P and K (74.33, 15.23 and $79.36 \mathrm{~kg} \mathrm{ha}^{-1}$, respectively) over bio-digester @ 20\% and was on par with borax @ $0.2 \%+$ $\mathrm{MgSO}_{4} @ 1 \%$ and vermiwash @ $20 \%$.

Among the organic treatment combinations, application of EC (1/3) + VC (1/3) + gliricidia GLM (1/3) equivalent to RDF with foliar spray of panchagavya @ 5\% recorded significantly higher uptake of $\mathrm{N}, \mathrm{P}_{2} \mathrm{O}_{5}$ and $\mathrm{K}_{2} \mathrm{O}$ (73.80, 15.16 and $78.4 \mathrm{~kg} \mathrm{ha}{ }^{-1}$, respectively) over other organic combinations and was on par with $(1 / 3)+\mathrm{VC}(1 / 3)+$ GLM (1/3) equivalent to RDF with borax @ $0.2 \%+$ $\mathrm{MgSO}_{4} @ 1 \%$ and RDF + FYM. Similar results were also observed by Sanjutha et al., (2008) and Tolanur (2008). Application of organics with foliar spray of panchagavya noticed enhanced biological efficiency of crop plants and created greater source and sink in the plant system (Boomathi et al., 2005). Integrated application of FYM @ $5 \mathrm{t} \mathrm{ha}^{-1}+$ $\mathrm{RDF}+$ panchagavya @ 5\% recorded significantly higher uptake of nitrogen, phosphorus and potassium (80.20, 17.39 and $81.82 \mathrm{~kg} \mathrm{ha}^{-1}$, respectively) over rest of the combinations but was on par with FYM @ $5 \mathrm{t}$ $\mathrm{ha}^{-1}+\mathrm{RDF}+$ borax @0.2\% + $\mathrm{MgSO}_{4} @ 1 \%$ and FYM @ $5 \mathrm{tha}^{-1}+\mathrm{RDF}+$ vermiwash @ $20 \%$. This might be due to higher total dry matter production in this treatment. Foliar application of boron accelerates the translocation of nitrogen compounds, increased the protein synthesis and stimulates fruiting and hastens the translocation of nitrogen and sugars thus improving fruiting resulted in higher biomass production and yield.

Among the nutrient management practices, integrated application of EC (1/3) + VC (1/3) + GLM (1/3) equivalent to RDF and EC (1/3) + VC (1/3) + GLM (1/3) equivalent to RDN recorded significantly higher organic carbon (5.6 and $5.5 \mathrm{~g} \mathrm{~kg}^{-1}$, respectively) over FYM @ $5 \mathrm{t} \mathrm{ha}^{-1}+\operatorname{RDF}\left(5.2 \mathrm{~g} \mathrm{~kg}^{-1}\right)$. It was higher by 7.96 per cent over FYM @ $5 \mathrm{t} \mathrm{ha}^{-1}+\mathrm{RDF}$. Lower organic carbon content was recorded in plots supplemented (Table 1) with RDF + FYM as compared to $100 \%$ organic manures application.

The foliar spray of panchagavya @ 5\% was recorded significantly higher soil organic carbon $\left(5.5 \mathrm{~g} \mathrm{~kg}^{-1}\right)$ over foliar spray of biodigester@20\%(5.3 $\left.\mathrm{g} \mathrm{kg}^{-1}\right)$ and was on par with borax @ 0.2\% + $\mathrm{MgSO}_{4} @ 1 \%$ (5.5 g $\left.\mathrm{kg}^{-1}\right)$. Among the different treatment combinations, application EC (1/3) + VC (1/3) + gliricidia GLM (1/3) equivalent to RDF with foliar spray of panchagavya @ 5\% recorded significantly higher soil organic carbon $(5.7 \mathrm{~g}$ $\left.\mathrm{kg}^{-1}\right)$ over RDF + FYM $\left(5.0 \mathrm{~g} \mathrm{~kg}^{-1}\right)$ and was on par with $\mathrm{EC}(1 / 3)+\mathrm{VC}(1 / 3)+$ gliricidia GLM (1/3) equivalent to RDF with foliar spray of borax @ $0.2 \%+\mathrm{MgSO}_{4} @ 1 \%$ and $\mathrm{EC}(1 / 3)+\mathrm{VC}(1 / 3)+$ gliricidia GLM $(1 / 3)$ equivalent to RDF with vermiwash @ 20\% (5.6 and $5.5 \mathrm{~g} \mathrm{~kg}^{-1}$, respectively).

The available soil $\mathrm{N}, \mathrm{P}_{2} \mathrm{O}_{5}$ and $\mathrm{K}_{2} \mathrm{O}$ varied significantly due to different nutrient management practices. Among the nutrient management practices, integrated application of EC $(1 / 3)+\mathrm{VC}(1 / 3)$ + gliricidia GLM (1/3) equivalent to $\mathrm{RDF}$ recorded (Table 2) significantly higher available soil $\mathrm{N}, \mathrm{P}_{2} \mathrm{O}_{5}$ and $\mathrm{K}_{2} \mathrm{O}\left(282.5,28.8,328.3 \mathrm{~kg} \mathrm{ha}^{-1}\right.$, respectively) over FYM @ $5 \mathrm{tha}^{-1}+\mathrm{RDF}$. 
Table.1 Bulk density in soil and uptake of N, P and K by cotton crop as influenced by organic manures, GLM, liquid organic manures and micronutrients

\begin{tabular}{|c|c|c|c|c|c|c|c|c|c|c|c|c|}
\hline \multirow[t]{2}{*}{ Treatment } & \multicolumn{4}{|c|}{2010} & \multicolumn{4}{|c|}{2011} & \multicolumn{4}{|c|}{ Pooled } \\
\hline & $\begin{array}{l}\text { Bulk density } \\
(\mathrm{g} / \mathrm{cc})\end{array}$ & $\mathrm{N}\left(\mathrm{kg} \mathrm{ha}^{-1}\right)$ & $\mathrm{P}\left(\mathrm{kg} \mathrm{ha}^{-1}\right)$ & $\mathrm{K}\left(\mathrm{kg} \mathrm{ha}^{-1}\right)$ & $\begin{array}{l}\text { Bulk density } \\
(\mathrm{g} / \mathrm{cc})\end{array}$ & $\mathrm{N}\left(\mathrm{kg} \mathrm{ha}^{-1}\right)$ & $\mathrm{P}\left(\mathrm{kg} \mathrm{ha}^{-1}\right)$ & $\mathrm{K}\left(\mathrm{kg} \mathrm{ha}^{-1}\right)$ & $\begin{array}{l}\text { Bulk density } \\
(\mathrm{g} / \mathrm{cc})\end{array}$ & $\mathrm{N}\left(\mathrm{kg} \mathrm{ha}^{-1}\right)$ & $\mathrm{P}\left(\mathrm{kg} \mathrm{ha}^{-1}\right)$ & $\mathrm{K}\left(\mathrm{kg} \mathrm{ha}^{-1}\right)$ \\
\hline \multicolumn{13}{|c|}{ Organic Manure (M) } \\
\hline $\mathrm{M}_{1}$ & $1.28 \mathrm{a}$ & $77.5 \mathrm{a}$ & $16.0 \mathrm{a}$ & $79.6 \mathrm{a}$ & $1.27 \mathrm{a}$ & $80.6 \mathrm{a}$ & $18.1 \mathrm{a}$ & $82.2 \mathrm{a}$ & $1.28 \mathrm{a}$ & $79.0 \mathrm{a}$ & 17.1a & $80.9 \mathrm{a}$ \\
\hline $\mathrm{M}_{2}$ & $1.27 \mathrm{a}$ & $66.3 \mathrm{c}$ & $11.6 \mathrm{c}$ & $75.6 \mathrm{~b}$ & $1.25 \mathrm{a}$ & $69.3 c$ & $13.3 \mathrm{c}$ & $77.9 \mathrm{~b}$ & $1.26 \mathrm{ab}$ & $67.8 \mathrm{c}$ & $12.5 \mathrm{c}$ & $76.8 \mathrm{c}$ \\
\hline $\mathrm{M}_{3}$ & $1.27 \mathrm{a}$ & $70.4 \mathrm{~b}$ & $13.5 b$ & $76.1 \mathrm{~b}$ & $1.24 \mathrm{a}$ & $74.6 \mathrm{~b}$ & $15.0 \mathrm{~b}$ & $78.5 b$ & $1.25 b$ & $72.5 b$ & $14.2 \mathrm{~b}$ & $77.3 b$ \\
\hline S.Em. \pm & 0.0082 & 0.30 & 0.11 & 0.20 & 0.011 & 0.26 & 0.10 & 0.17 & 0.0082 & 0.28 & 0.10 & 0.10 \\
\hline \multicolumn{13}{|c|}{ Foliar spray of liquid manures + micronutrients (L) } \\
\hline $\mathrm{L}_{1}$ & $1.27 \mathrm{a}$ & $72.4 \mathrm{a}$ & $14.2 \mathrm{a}$ & $78.1 \mathrm{a}$ & $1.25 \mathrm{a}$ & $76.2 \mathrm{a}$ & $16.2 \mathrm{a}$ & $80.5 \mathrm{a}$ & $1.26 \mathrm{a}$ & $74.3 \mathrm{a}$ & $15.2 \mathrm{a}$ & $79.3 \mathrm{a}$ \\
\hline $\mathrm{L}_{2}$ & $1.26 \mathrm{a}$ & $69.6 \mathrm{~b}$ & $12.7 \mathrm{~b}$ & $75.6 \mathrm{c}$ & $1.23 \mathrm{a}$ & $72.3 \mathrm{~d}$ & $14.2 \mathrm{~b}$ & $77.9 \mathrm{c}$ & $1.27 \mathrm{a}$ & $71.0 \mathrm{~b}$ & $13.5 b$ & $76.8 \mathrm{~d}$ \\
\hline $\mathrm{L}_{3}$ & $1.27 \mathrm{a}$ & $71.3 \mathrm{a}$ & $13.7 \mathrm{a}$ & $76.7 \mathrm{bc}$ & $1.26 \mathrm{a}$ & $74.7 \mathrm{c}$ & $15.4 \mathrm{a}$ & $79.0 \mathrm{bc}$ & $1.27 \mathrm{a}$ & $73.0 \mathrm{a}$ & $14.5 \mathrm{a}$ & $77.8 \mathrm{c}$ \\
\hline $\mathrm{L}_{4}$ & $1.27 \mathrm{a}$ & 71.6a & $13.7 \mathrm{a}$ & 77.2ab & $1.25 \mathrm{a}$ & $75.1 \mathrm{c}$ & $15.5 \mathrm{a}$ & $79.8 \mathrm{ab}$ & $1.26 \mathrm{a}$ & $73.3 \mathrm{a}$ & $14.6 \mathrm{a}$ & $78.5 b$ \\
\hline $\mathrm{L}_{5}$ & $1.27 \mathrm{a}$ & $72.2 \mathrm{a}$ & $14.1 \mathrm{a}$ & 77.1ab & $1.25 \mathrm{a}$ & $75.7 \mathrm{~b}$ & $16.0 \mathrm{a}$ & $80.4 \mathrm{a}$ & $1.26 \mathrm{a}$ & $74.0 \mathrm{a}$ & $15.1 \mathrm{a}$ & $79.1 \mathrm{ab}$ \\
\hline S.Em. \pm & 0.011 & 0.43 & 0.28 & 0.42 & 0.014 & 0.14 & 0.26 & 0.39 & 0.0105 & 0.42 & 0.27 & 0.21 \\
\hline \multicolumn{13}{|c|}{ Interactions (MXL) } \\
\hline $\mathrm{M}_{1} \mathrm{~L}_{1}$ & $1.28 \mathrm{ab}$ & $78.5 \mathrm{a}$ & $16.1 \mathrm{a}$ & $80.5 \mathrm{a}$ & $1.26 \mathrm{a}-\mathrm{d}$ & $81.9 \mathrm{a}$ & $18.6 \mathrm{a}$ & $83.0 \mathrm{a}$ & $1.27 \mathrm{ab}$ & $80.2 \mathrm{a}$ & $17.3 \mathrm{a}$ & $81.8 \mathrm{a}$ \\
\hline $\mathrm{M}_{1} \mathrm{~L}_{2}$ & $1.27 \mathrm{a}-\mathrm{c}$ & $76.0 \mathrm{~b}$ & $15.8 \mathrm{a}$ & $78.3 \mathrm{~b}-\mathrm{e}$ & $1.27 \mathrm{ab}$ & $78.5 b c$ & $17.7 \mathrm{ab}$ & $80.9 b-d$ & $1.27 \mathrm{ab}$ & $77.3 b c$ & $16.7 \mathrm{ab}$ & $79.6 \mathrm{~cd}$ \\
\hline $\mathrm{M}_{1} \mathrm{~L}_{3}$ & $1.28 \mathrm{ab}$ & $77.2 \mathrm{ab}$ & $16.0 \mathrm{a}$ & 79.1a-d & $1.27 \mathrm{a}-\mathrm{d}$ & $80.2 \mathrm{ab}$ & $18.0 \mathrm{ab}$ & $81.5 \mathrm{a}-\mathrm{c}$ & $1.27 \mathrm{ab}$ & 78.7ab & 17.0ab & $80.3 b c$ \\
\hline $\mathrm{M}_{1} \mathrm{~L}_{4}$ & $1.28 \mathrm{a}$ & 77.6ab & $16.0 \mathrm{a}$ & $79.9 \mathrm{a}-\mathrm{c}$ & $1.27 \mathrm{a}-\mathrm{c}$ & $80.8 \mathrm{a}$ & $18.2 \mathrm{a}$ & $82.5 \mathrm{ab}$ & $1.28 \mathrm{a}$ & 79.2ab & $17.1 \mathrm{a}$ & $81.2 \mathrm{ab}$ \\
\hline $\mathrm{M}_{1} \mathrm{~L}_{5}$ & $1.28 \mathrm{a}$ & $78.3 \mathrm{ab}$ & $16.1 \mathrm{a}$ & $80.3 \mathrm{ab}$ & $1.26 \mathrm{a}-\mathrm{e}$ & $81.4 \mathrm{a}$ & $18.3 \mathrm{a}$ & $83.0 \mathrm{a}$ & $1.27 \mathrm{ab}$ & $79.8 \mathrm{a}$ & $17.2 \mathrm{a}$ & $81.6 \mathrm{a}$ \\
\hline $\mathrm{M}_{2} \mathrm{~L}_{1}$ & $1.27 b c$ & $67.2 \mathrm{f}$ & $12.1 \mathrm{~cd}$ & 76.6e-g & $1.24 \mathrm{c}-\mathrm{e}$ & $70.7 \mathrm{fg}$ & 14.1ef & 79.1d-f & $1.26 \mathrm{~d}-\mathrm{f}$ & $68.9 \mathrm{fg}$ & 13.1ef & $77.9 \mathrm{f}-\mathrm{h}$ \\
\hline $\mathrm{M}_{2} \mathrm{~L}_{2}$ & $1.27 b c$ & $64.4 \mathrm{~g}$ & $10.5 \mathrm{e}$ & $74.2 \mathrm{i}$ & $1.26 \mathrm{a}-\mathrm{d}$ & $66.6 \mathrm{~h}$ & $11.0 \mathrm{~g}$ & $76.3 \mathrm{~g}$ & $1.27 \mathrm{a}-\mathrm{d}$ & $65.5 \mathrm{~h}$ & $11.1 \mathrm{~g}$ & $75.3 \mathrm{~h}$ \\
\hline $\mathrm{M}_{2} \mathrm{~L}_{3}$ & $1.27 \mathrm{a}-\mathrm{c}$ & $66.4 \mathrm{fg}$ & $11.7 \mathrm{de}$ & $75.0 \mathrm{~g}-\mathrm{i}$ & $1.26 \mathrm{a}-\mathrm{d}$ & $69.3 \mathrm{~g}$ & $13.2 \mathrm{f}$ & $77.3 \mathrm{fg}$ & $1.27 \mathrm{a}-\mathrm{c}$ & $67.8 \mathrm{~g}$ & $12.5 \mathrm{f}$ & 76.2ij \\
\hline $\mathrm{M}_{2} \mathrm{~L}_{4}$ & $1.26 \mathrm{c}$ & $66.6 f$ & $11.7 \mathrm{de}$ & $75.7 \mathrm{~g}-\mathrm{i}$ & $1.25 \mathrm{a}-\mathrm{e}$ & $69.8 \mathrm{fg}$ & $13.4 \mathrm{f}$ & $78.1 \mathrm{e}-\mathrm{g}$ & $1.26 \mathrm{~b}-\mathrm{e}$ & $68.2 \mathrm{fg}$ & $12.6 \mathrm{f}$ & 76.9hi \\
\hline $\mathrm{M}_{2} \mathrm{~L}_{5}$ & $1.27 \mathrm{a}-\mathrm{c}$ & 67.1f & $12.1 \mathrm{~cd}$ & $76.5 \mathrm{e}-\mathrm{h}$ & $1.25 \mathrm{~b}-\mathrm{e}$ & $70.2 \mathrm{fg}$ & 14.1ef & $78.9 \mathrm{~d}-\mathrm{f}$ & $1.26 \mathrm{~b}-\mathrm{e}$ & $68.7 \mathrm{fg}$ & 13.1ef & 77.7f-h \\
\hline $\mathrm{M}_{3} \mathrm{~L}_{1}$ & $1.26 \mathrm{c}$ & $71.5 \mathrm{~cd}$ & $14.3 b$ & 77.1d-g & $1.23 \mathrm{e}$ & 76.0de & $15.9 \mathrm{~cd}$ & $79.5 c-e$ & $1.25 \mathrm{f}$ & 73.8de & $15.1 \mathrm{~cd}$ & $78.3 \mathrm{ef}$ \\
\hline $\mathrm{M}_{3} \mathrm{~L}_{2}$ & $1.27 \mathrm{a}-\mathrm{c}$ & $68.4 \mathrm{ef}$ & $11.9 \mathrm{~d}$ & 74.4hi & $1.25 \mathrm{~b}-\mathrm{e}$ & $71.8 \mathrm{f}$ & $13.2 \mathrm{f}$ & $76.6 \mathrm{~g}$ & $1.26 \mathrm{c}-\mathrm{f}$ & $70.1 \mathrm{f}$ & $12.6 \mathrm{f}$ & $75.5 \mathrm{j}$ \\
\hline $\mathrm{M}_{3} \mathrm{~L}_{3}$ & $1.27 b c$ & 70.4de & $13.4 \mathrm{bc}$ & $75.8 \mathrm{f}-\mathrm{i}$ & $1.25 \mathrm{a}-\mathrm{e}$ & 74.6e & 14.9de & $78.2 \mathrm{e}-\mathrm{g}$ & $1.26 \mathrm{~b}-\mathrm{e}$ & $72.5 \mathrm{e}$ & $14.2 \mathrm{de}$ & $77.0 \mathrm{~g}-\mathrm{i}$ \\
\hline $\mathrm{M}_{3} \mathrm{~L}_{4}$ & $1.26 \mathrm{c}$ & $70.5 \mathrm{de}$ & $13.5 b c$ & $76.3 e-i$ & $1.24 \mathrm{de}$ & $74.8 \mathrm{de}$ & 15.0de & $78.8 \mathrm{ef}$ & $1.25 \mathrm{ef}$ & $72.6 \mathrm{e}$ & $14.2 \mathrm{de}$ & $77.5 \mathrm{f}-\mathrm{h}$ \\
\hline $\mathrm{M}_{3} \mathrm{~L}_{5}$ & $1.27 \mathrm{a}-\mathrm{c}$ & $71.2 \mathrm{~d}$ & $14.2 \mathrm{~b}$ & $76.8 \mathrm{e}-\mathrm{g}$ & $1.24 \mathrm{de}$ & 75.6de & $15.8 \mathrm{~cd}$ & $79.3 \mathrm{~d}-\mathrm{f}$ & $1.26 \mathrm{~d}-\mathrm{f}$ & 73.4de & $15.0 \mathrm{~cd}$ & 78.1e-g \\
\hline $\mathrm{C}_{1}$ & $1.28 \mathrm{ab}$ & 73.0fc & $14.7 \mathrm{ab}$ & $78.0 \mathrm{c}-\mathrm{f}$ & $1.28 \mathrm{a}$ & $77.1 \mathrm{~cd}$ & $16.7 b c$ & $80.0 \mathrm{c}-\mathrm{e}$ & $1.28 \mathrm{a}$ & $75.3 \mathrm{~cd}$ & $15.7 b c$ & 79.0de \\
\hline S.Em. \pm & 0.0033 & 0.72 & 0.46 & 0.67 & 0.0082 & 0.71 & 0.43 & 0.63 & 0.0033 & 0.69 & 0.43 & 0.35 \\
\hline
\end{tabular}

Note: EC- Enriched compost; C- Compost; VC - Vermicompost; $\mathrm{M}_{1-} \mathrm{RDF}-80: 40: 40 \mathrm{NPK} k g$ ha $^{-1}+\mathrm{FYM} @ 5$ t ha ${ }^{-1} ; \mathrm{M}_{2}-\mathrm{EC}(1 / 3)+\mathrm{VC}(1 / 3)+\mathrm{GLM}(1 / 3)$ equi.to RDN;

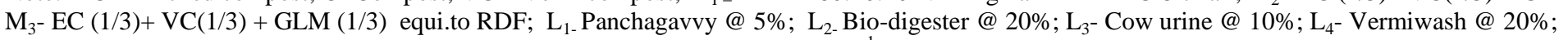

$\mathrm{L}_{5}$ - borax @ 0.2\% + $\mathrm{MgSO}_{4} @ 1 \% ; \mathrm{C}_{1}-\mathrm{RDF}-80: 40: 40 \mathrm{NPK} \mathrm{kg}+\mathrm{FYM} @ 5 \mathrm{t} \mathrm{ha}^{-1}$ 
Table.2 Organic carbon and available $\mathrm{N}, \mathrm{P}_{2} \mathrm{O}_{5}$ and $\mathrm{K}_{2} \mathrm{O}$ in soil as influenced by organic manures, GLM, liquid organic manures and micronutrients

\begin{tabular}{|c|c|c|c|c|c|c|c|c|c|c|c|c|}
\hline \multirow[t]{2}{*}{ Treatment } & \multicolumn{4}{|c|}{2010} & \multicolumn{4}{|c|}{2011} & \multicolumn{4}{|c|}{ Pooled } \\
\hline & $\underset{\left(\mathrm{g} \mathrm{kg}^{-1}\right)}{\mathbf{O C}}$ & $\begin{array}{c}\mathrm{N} \\
\left(\mathrm{kg} \mathrm{ha}^{-1}\right)\end{array}$ & $\begin{array}{c}\mathrm{P}_{2} \mathrm{O}_{5} \\
\left(\mathrm{~kg} \mathrm{ha}^{-1}\right)\end{array}$ & $\begin{array}{c}\mathrm{K}_{2} \mathrm{O} \\
\left(\mathrm{kg} \mathrm{ha}^{-1}\right)\end{array}$ & $\underset{\left.\left(\mathrm{g} \mathrm{kg}^{-1}\right)\right)}{\mathbf{O C}}$ & $\begin{array}{c}\mathrm{N} \\
\left(\mathrm{kg} \mathrm{ha}^{-1}\right)\end{array}$ & $\begin{array}{c}\mathrm{P}_{2} \mathrm{O}_{5} \\
\left(\mathrm{~kg} \mathrm{ha}^{-1}\right)\end{array}$ & $\begin{array}{c}\mathrm{K}_{2} \mathrm{O} \\
\left(\mathrm{kg} \mathrm{ha}^{-1}\right)\end{array}$ & $\underset{\left(\mathrm{g} \mathrm{kg}^{-1}\right)}{\mathbf{O C}}$ & $N\left(k h^{-1}\right)$ & $\begin{array}{c}\mathrm{P}_{2} \mathrm{O}_{5} \\
\left(\mathrm{~kg} \mathrm{ha}^{-1}\right)\end{array}$ & $\begin{array}{c}\mathrm{K}_{2} \mathrm{O} \\
\left(\mathrm{kg} \mathrm{ha}^{-1}\right)\end{array}$ \\
\hline \multicolumn{13}{|c|}{ Organic Manure (M) } \\
\hline $\mathrm{M}_{1}$ & $5.1 \mathrm{~b}$ & $270.8 \mathrm{c}$ & $25.6 \mathrm{c}$ & $311.4 \mathrm{~b}$ & $5.3 \mathrm{~b}$ & $275.5 \mathrm{c}$ & $27.0 \mathrm{c}$ & $317.6 \mathrm{~b}$ & $5.2 \mathrm{~b}$ & $273.2 \mathrm{c}$ & $26.3 \mathrm{c}$ & $314.5 b$ \\
\hline $\mathrm{M}_{2}$ & $5.3 \mathrm{ab}$ & $275.5 b$ & $26.2 \mathrm{~b}$ & $323.4 \mathrm{a}$ & $5.6 \mathrm{a}$ & $279.8 \mathrm{~b}$ & $28.3 b$ & $330.5 \mathrm{a}$ & $5.5 \mathrm{a}$ & $277.6 b$ & $27.3 b$ & $327.0 \mathrm{a}$ \\
\hline $\mathrm{M}_{3}$ & $5.4 \mathrm{a}$ & $277.7 \mathrm{a}$ & $27.6 \mathrm{a}$ & $324.7 \mathrm{a}$ & $5.7 \mathrm{a}$ & $287.3 \mathrm{a}$ & $30.1 \mathrm{a}$ & $331.8 \mathrm{a}$ & $5.6 \mathrm{a}$ & $282.5 \mathrm{a}$ & $28.8 \mathrm{a}$ & $328.3 \mathrm{a}$ \\
\hline S.Em. \pm & 0.0058 & 0.35 & 0.13 & 0.49 & 0.0058 & 0.30 & 0.15 & 0.81 & 0.0041 & 0.29 & 0.08 & 0.56 \\
\hline \multicolumn{13}{|c|}{ Foliar spray of liquid manures + micronutrients (L) } \\
\hline $\mathrm{L}_{1}$ & $5.3 \mathrm{a}$ & $276.1 \mathrm{a}$ & $27.3 \mathrm{a}$ & $320.9 \mathrm{a}$ & $5.7 \mathrm{a}$ & $282.7 \mathrm{a}$ & $29.4 \mathrm{a}$ & $328.0 \mathrm{a}$ & $5.5 \mathrm{a}$ & $279.4 a$ & $28.3 \mathrm{a}$ & $324.4 \mathrm{a}$ \\
\hline $\mathrm{L}_{2}$ & $5.2 \mathrm{~b}$ & $272.9 \mathrm{~b}$ & $25.4 \mathrm{~b}$ & $318.8 \mathrm{~b}$ & $5.4 \mathrm{c}$ & $278.2 \mathrm{~b}$ & $26.8 \mathrm{~b}$ & $325.3 \mathrm{c}$ & $5.3 \mathrm{~d}$ & $275.6 b$ & $26.1 \mathrm{~b}$ & $322.1 b$ \\
\hline $\mathrm{L}_{3}$ & $5.3 \mathrm{ab}$ & $274.4 \mathrm{ab}$ & $26.2 \mathrm{ab}$ & $319.2 \mathrm{ab}$ & $5.5 \mathrm{bc}$ & $280.5 \mathrm{ab}$ & $28.3 \mathrm{a}$ & $325.9 \mathrm{bc}$ & $5.4 \mathrm{~cd}$ & $277.5 \mathrm{a}$ & $27.3 \mathrm{a}$ & $322.6 \mathrm{ab}$ \\
\hline $\mathrm{L}_{4}$ & $5.3 \mathrm{ab}$ & $274.6 \mathrm{ab}$ & $26.4 a b$ & $319.7 \mathrm{ab}$ & $5.5 \mathrm{a}-\mathrm{c}$ & $280.9 \mathrm{ab}$ & $28.5 \mathrm{a}$ & $326.5 a-c$ & $5.4 \mathrm{bc}$ & $277.8 \mathrm{a}$ & $27.5 \mathrm{a}$ & $323.1 \mathrm{ab}$ \\
\hline $\mathrm{L}_{5}$ & $5.3 \mathrm{a}$ & $275.3 a$ & $27.1 \mathrm{a}$ & $320.7 \mathrm{a}$ & $5.6 \mathrm{ab}$ & $282.1 \mathrm{a}$ & $29.2 \mathrm{a}$ & $327.5 \mathrm{ab}$ & $5.5 \mathrm{ab}$ & $278.7 \mathrm{a}$ & $28.1 \mathrm{a}$ & $324.1 \mathrm{a}$ \\
\hline S.Em. \pm & 0.0043 & 0.68 & 0.42 & 0.56 & 0.0065 & 0.56 & 0.38 & 0.54 & 0.0030 & 0.62 & 0.38 & 0.47 \\
\hline \multicolumn{13}{|c|}{ Interactions (MXL) } \\
\hline $\mathrm{M}_{1} \mathrm{~L}_{1}$ & $5.2 \mathrm{c}-\mathrm{f}$ & 272.7d-f & $26.5 \mathrm{a}-\mathrm{d}$ & $312.7 \mathrm{c}$ & $5.4 \mathrm{c}-\mathrm{e}$ & $277.2 \mathrm{c}-\mathrm{f}$ & $27.8 \mathrm{c}-\mathrm{e}$ & $319.0 \mathrm{~b}$ & $5.3 \mathrm{f}-\mathrm{i}$ & 275.0de & $27.2 \mathrm{~b}-\mathrm{e}$ & $315.9 \mathrm{c}$ \\
\hline $\mathrm{M}_{1} \mathrm{~L}_{2}$ & $5.0 \mathrm{ef}$ & $269.7 \mathrm{gh}$ & $24.2 \mathrm{de}$ & $310.0 \mathrm{c}$ & $5.2 \mathrm{de}$ & $273.8 \mathrm{fg}$ & $25.1 \mathrm{fg}$ & $316.0 \mathrm{~b}$ & $5.1 \mathrm{ij}$ & $271.8 \mathrm{ef}$ & $24.6 f g$ & $313.0 \mathrm{c}$ \\
\hline $\mathrm{M}_{1} \mathrm{~L}_{3}$ & $5.1 \mathrm{~d}-\mathrm{f}$ & $270.3 \mathrm{f}-\mathrm{h}$ & $25.4 \mathrm{~b}-\mathrm{e}$ & $310.4 \mathrm{c}$ & $5.2 \mathrm{de}$ & $274.6 \mathrm{fg}$ & $27.1 \mathrm{~d}-\mathrm{f}$ & $316.4 b$ & $5.2 \mathrm{~h}-\mathrm{j}$ & $272.5 \mathrm{e}$ & $26.2 \mathrm{ef}$ & $313.4 \mathrm{c}$ \\
\hline $\mathrm{M}_{1} \mathrm{~L}_{4}$ & $5.1 \mathrm{~d}-\mathrm{f}$ & $270.3 \mathrm{f}-\mathrm{h}$ & $25.5 \mathrm{~b}-\mathrm{e}$ & $311.5 \mathrm{c}$ & $5.3 c-e$ & $275.2 \mathrm{e}-\mathrm{g}$ & $27.2 \mathrm{~d}-\mathrm{f}$ & $317.7 \mathrm{~b}$ & $5.2 \mathrm{~g}-\mathrm{i}$ & $272.7 \mathrm{e}$ & $26.4 \mathrm{~d}-\mathrm{f}$ & $314.6 \mathrm{c}$ \\
\hline $\mathrm{M}_{1} \mathrm{~L}_{5}$ & $5.2 \mathrm{~b}-\mathrm{f}$ & $271.2 \mathrm{e}-\mathrm{h}$ & $26.3 a-d$ & $312.6 c$ & $5.4 \mathrm{~b}-\mathrm{e}$ & $276.8 \mathrm{c}-\mathrm{f}$ & $27.7 \mathrm{c}-\mathrm{e}$ & $318.8 \mathrm{~b}$ & $5.3 \mathrm{e}-\mathrm{i}$ & $274.0 \mathrm{e}$ & $27.0 c-e$ & $315.7 \mathrm{c}$ \\
\hline $\mathrm{M}_{2} \mathrm{~L}_{1}$ & $5.4 a-d$ & $276.2 \mathrm{a}-\mathrm{d}$ & $27.0 \mathrm{a}-\mathrm{c}$ & $323.8 \mathrm{ab}$ & $5.8 \mathrm{ab}$ & $281.6 b c$ & 29.1a-d & $331.3 \mathrm{a}$ & $5.6 \mathrm{a}-\mathrm{c}$ & $278.9 \mathrm{c}$ & $28.1 \mathrm{a}-\mathrm{e}$ & $327.5 \mathrm{ab}$ \\
\hline $\mathrm{M}_{2} \mathrm{~L}_{2}$ & $5.2 b-f$ & $274.0 \mathrm{c}-\mathrm{f}$ & $25.2 \mathrm{c}-\mathrm{e}$ & $322.8 b$ & $5.5 \mathrm{a}-\mathrm{d}$ & 275.9d-f & $26.6 \mathrm{e}-\mathrm{g}$ & $329.6 a$ & $5.4 \mathrm{~d}-\mathrm{h}$ & 275.0de & $25.9 \mathrm{f}-\mathrm{g}$ & $326.2 \mathrm{bab}$ \\
\hline $\mathrm{M}_{2} \mathrm{~L}_{3}$ & $5.3 \mathrm{a}-\mathrm{d}$ & $275.3 a-d$ & $25.9 \mathrm{a}-\mathrm{d}$ & $323.2 \mathrm{ab}$ & $5.5 \mathrm{a}-\mathrm{d}$ & $280.0 c-e$ & $28.1 \mathrm{c}-\mathrm{e}$ & $330.2 \mathrm{a}$ & $5.4 \mathrm{c}-\mathrm{g}$ & $277.7 \mathrm{~cd}$ & $27.0 \mathrm{c}-\mathrm{e}$ & $326.7 \mathrm{ab}$ \\
\hline $\mathrm{M}_{2} \mathrm{~L}_{4}$ & $5.3 \mathrm{a}-\mathrm{d}$ & $275.8 \mathrm{a}-\mathrm{d}$ & 26.1a-d & $323.3 \mathrm{ab}$ & $5.6 a-c$ & $280.6 b-d$ & 28.6b-e & $330.5 \mathrm{a}$ & $5.5 \mathrm{~b}-\mathrm{e}$ & $278.2 \mathrm{c}$ & $27.3 \mathrm{~b}-\mathrm{e}$ & $326.9 \mathrm{ab}$ \\
\hline $\mathrm{M}_{2} \mathrm{~L}_{5}$ & $5.4 \mathrm{a}-\mathrm{d}$ & $276.0 \mathrm{a}-\mathrm{d}$ & $26.9 \mathrm{a}-\mathrm{c}$ & $323.9 \mathrm{ab}$ & $5.8 \mathrm{ab}$ & $281.1 b c$ & $29.1 \mathrm{a}-\mathrm{d}$ & $331.1 \mathrm{a}$ & $5.6 \mathrm{a}-\mathrm{c}$ & $278.5 \mathrm{c}$ & $28.0 \mathrm{a}-\mathrm{e}$ & $327.5 \mathrm{ab}$ \\
\hline $\mathrm{M}_{3} \mathrm{~L}_{1}$ & $5.5 \mathrm{a}$ & $279.4 a$ & $28.3 \mathrm{a}$ & $326.4 \mathrm{a}$ & $5.9 \mathrm{a}$ & $289.4 \mathrm{a}$ & $31.2 \mathrm{a}$ & $333.6 \mathrm{a}$ & $5.7 \mathrm{a}$ & $284.4 \mathrm{a}$ & $29.7 \mathrm{a}$ & $330.0 \mathrm{a}$ \\
\hline $\mathrm{M}_{3} \mathrm{~L}_{2}$ & $5.3 \mathrm{a}-\mathrm{e}$ & $275.0 \mathrm{~b}-\mathrm{e}$ & $26.8 \mathrm{a}-\mathrm{c}$ & $323.5 \mathrm{ab}$ & $5.6 \mathrm{a}-\mathrm{d}$ & $285.0 \mathrm{ab}$ & $28.7 b-\mathrm{e}$ & $330.5 \mathrm{a}$ & $5.4 \mathrm{~b}-\mathrm{f}$ & $280.0 \mathrm{bc}$ & $27.8 \mathrm{a}-\mathrm{e}$ & $327.0 \mathrm{ab}$ \\
\hline $\mathrm{M}_{3} \mathrm{~L}_{3}$ & $5.4 \mathrm{a}-\mathrm{d}$ & $277.6 a-c$ & $27.4 a-c$ & $324.2 \mathrm{ab}$ & $5.7 \mathrm{a}-\mathrm{c}$ & $286.9 \mathrm{a}$ & $29.7 a-c$ & $331.2 \mathrm{a}$ & $5.5 \mathrm{a}-\mathrm{d}$ & $282.2 \mathrm{ab}$ & $28.5 \mathrm{a}-\mathrm{d}$ & $327.7 \mathrm{ab}$ \\
\hline $\mathrm{M}_{3} \mathrm{~L}_{4}$ & $5.4 \mathrm{a}-\mathrm{c}$ & 277.9a-c & $27.7 \mathrm{ab}$ & $324.1 \mathrm{ab}$ & $5.6 \mathrm{a}-\mathrm{c}$ & $287.0 \mathrm{a}$ & $29.9 a-c$ & $331.4 \mathrm{a}$ & $5.5 \mathrm{a}-\mathrm{d}$ & $282.5 \mathrm{ab}$ & $28.8 \mathrm{a}-\mathrm{c}$ & $327.7 \mathrm{ab}$ \\
\hline $\mathrm{M}_{3} \mathrm{~L}_{5}$ & $5.5 \mathrm{ab}$ & $278.6 \mathrm{ab}$ & $28.0 \mathrm{a}$ & $325.5 \mathrm{ab}$ & $5.8 \mathrm{ab}$ & $288.4 \mathrm{a}$ & $30.8 \mathrm{ab}$ & $332.7 \mathrm{a}$ & $5.6 a b$ & $283.5 \mathrm{a}$ & $29.4 \mathrm{ab}$ & $329.1 \mathrm{ab}$ \\
\hline $\mathrm{C}_{1}$ & $4.9 \mathrm{f}$ & $267.5 \mathrm{~h}$ & $23.5 \mathrm{e}$ & $291.7 d$ & $5.1 \mathrm{e}$ & $270.9 \mathrm{~g}$ & $24.6 \mathrm{~g}$ & $290.2 \mathrm{c}$ & $5.0 \mathrm{j}$ & $269.2 \mathrm{e}$ & $24.1 \mathrm{~g}$ & $291.0 \mathrm{~d}$ \\
\hline S.Em. \pm & 0.0088 & 1.28 & 0.71 & 1.0 .3 & 0.011 & 1.52 & 0.68 & 1.42 & 0.0058 & 1.05 & 0.66 & 1.00 \\
\hline
\end{tabular}

Note: EC- Enriched compost; C- Compost; VC - Vermicompost ; $\mathrm{M}_{1-} \mathrm{RDF}-80: 40: 40 \mathrm{NPK} \mathrm{kg} \mathrm{ha}^{-1}+\mathrm{FYM} @ 5$ t ha ${ }^{-1} ; \mathrm{M}_{2}-\mathrm{EC}(1 / 3)+\mathrm{VC}(1 / 3)+\mathrm{GLM}(1 / 3)$ equi.to RDN ;

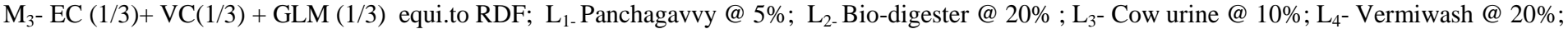

$\mathrm{L}_{5}$ - borax @ 0.2\% + $\mathrm{MgSO}_{4} @ 1 \% ; \mathrm{C}_{1}-\mathrm{RDF}-80: 40: 40 \mathrm{NPK} \mathrm{kg}+\mathrm{FYM} @ 5 \mathrm{tha}^{-1}$ 
Table.3 Actinomycetes (cfuX102 / g of soil) and $\mathrm{N}_{2}$ (cfuX103 / g of soil) fixers population observed in cotton soil as influenced by organic manures, GLM, liquid organic manures and micronutrients

\begin{tabular}{|c|c|c|c|c|c|c|c|c|c|c|c|c|}
\hline \multirow[t]{2}{*}{ Treatment } & \multicolumn{4}{|c|}{2010} & \multicolumn{4}{|c|}{2011} & \multicolumn{4}{|c|}{ Pooled } \\
\hline & $\begin{array}{l}\text { Actinomycetes } \\
\text { at } 60 \text { DAS }\end{array}$ & $\begin{array}{l}\text { Actinomycetes at } \\
90 \text { DAS }\end{array}$ & $\begin{array}{l}N_{2} \text { fixers } \\
\text { at } 60 \text { DAS }\end{array}$ & $\begin{array}{l}\mathrm{N}_{2} \text { fixers at } \\
90 \text { DAS }\end{array}$ & $\begin{array}{l}\text { Actinomycete } \\
\text { s at } 60 \text { DAS }\end{array}$ & $\begin{array}{l}\text { Actinomycet } \\
\text { es at } 90 \text { DAS }\end{array}$ & $\begin{array}{l}\mathrm{N}_{2} \text { fixers at } \\
60 \text { DAS }\end{array}$ & $\begin{array}{l}\mathrm{N}_{2} \text { fixers at } \\
90 \text { DAS }\end{array}$ & $\begin{array}{l}\text { Actinomycete } \\
\text { s at } 60 \text { DAS }\end{array}$ & $\begin{array}{c}\text { Actinomycet } \\
\text { es at } 90 \\
\text { DAS }\end{array}$ & $\begin{array}{l}\mathrm{N}_{2} \text { fixers at } \\
60 \text { DAS }\end{array}$ & $\begin{array}{l}N_{2} \text { fixers at } \\
90 \text { DAS }\end{array}$ \\
\hline \multicolumn{13}{|c|}{ Organic Manure (M) } \\
\hline $\mathrm{M}_{1}$ & $34.40 \mathrm{c}$ & $32.13 c$ & $23.93 \mathrm{c}$ & $22.33 c$ & $36.83 \mathrm{c}$ & $34.68 \mathrm{c}$ & $25.79 \mathrm{c}$ & $23.75 \mathrm{c}$ & $35.62 \mathrm{c}$ & $33.41 \mathrm{c}$ & $24.86 \mathrm{c}$ & $23.04 \mathrm{c}$ \\
\hline $\mathrm{M}_{2}$ & $36.67 \mathrm{~b}$ & $34.27 \mathrm{~b}$ & $27.60 \mathrm{~b}$ & $25.00 \mathrm{~b}$ & $38.78 b$ & $36.58 b$ & $30.81 \mathrm{~b}$ & $28.46 b$ & $37.72 b$ & $35.43 b$ & $29.21 b$ & $26.73 b$ \\
\hline $\mathrm{M}_{3}$ & $38.27 \mathrm{a}$ & $35.67 \mathrm{a}$ & $32.00 \mathrm{a}$ & $27.47 \mathrm{a}$ & $41.03 \mathrm{a}$ & $38.73 a$ & $34.31 \mathrm{a}$ & $31.57 \mathrm{a}$ & $39.65 a$ & $37.20 \mathrm{a}$ & $33.15 a$ & $29.52 \mathrm{a}$ \\
\hline S.Em. \pm & 0.239 & 0.154 & 0.539 & 0.353 & 0.182 & 0.136 & 0.511 & 0.235 & 0.174 & 0.143 & 0.335 & 0.294 \\
\hline \multicolumn{13}{|c|}{ Foliar spray of liquid manures + micronutrients (L) } \\
\hline $\mathrm{L}_{1}$ & $36.67 \mathrm{a}$ & $34.44 a$ & $30.56 a$ & $28.78 \mathrm{a}$ & $39.02 \mathrm{a}$ & $36.73 \mathrm{ab}$ & $34.06 \mathrm{a}$ & $32.07 \mathrm{a}$ & $37.85 \mathrm{a}$ & 35.59ab & $32.31 \mathrm{a}$ & $30.42 \mathrm{a}$ \\
\hline $\mathrm{L}_{2}$ & $35.89 \mathrm{a}$ & $33.33 b$ & $24.56 \mathrm{~b}$ & $21.67 \mathrm{c}$ & $38.49 \mathrm{a}$ & $35.61 \mathrm{~b}$ & $26.72 \mathrm{c}$ & $23.92 \mathrm{c}$ & $37.19 \mathrm{a}$ & $34.47 \mathrm{~b}$ & $25.64 \mathrm{c}$ & $22.79 \mathrm{c}$ \\
\hline $\mathrm{L}_{3}$ & $36.11 \mathrm{a}$ & $33.78 \mathrm{ab}$ & $25.56 \mathrm{~b}$ & $22.33 c$ & $38.78 \mathrm{a}$ & $36.73 \mathrm{ab}$ & $27.90 \mathrm{c}$ & $25.19 \mathrm{c}$ & $37.45 \mathrm{a}$ & $35.25 \mathrm{ab}$ & $26.73 c$ & $23.76 \mathrm{c}$ \\
\hline $\mathrm{L}_{4}$ & $37.11 \mathrm{a}$ & $34.44 \mathrm{a}$ & $28.56 \mathrm{a}$ & $25.33 b$ & $39.00 \mathrm{a}$ & $37.29 \mathrm{a}$ & $30.81 \mathrm{~b}$ & $28.62 b$ & $38.06 \mathrm{a}$ & $35.87 \mathrm{a}$ & $29.68 b$ & $26.98 b$ \\
\hline $\mathrm{L}_{5}$ & $36.44 \mathrm{a}$ & $34.11 \mathrm{ab}$ & $30.00 \mathrm{a}$ & $26.56 \mathrm{~b}$ & $39.11 \mathrm{a}$ & $36.95 \mathrm{ab}$ & $32.03 b$ & $29.85 b$ & $37.78 \mathrm{a}$ & $35.53 \mathrm{ab}$ & $31.02 \mathrm{ab}$ & $28.20 \mathrm{~b}$ \\
\hline S.Em. \pm & 0.45 & 0.329 & 0.779 & 0.729 & 0.385 & 0.438 & 0.625 & 0.761 & 0.278 & 0.37 & 0.504 & 0.732 \\
\hline \multicolumn{13}{|c|}{ Interactions (MXL) } \\
\hline $\mathrm{M}_{1} \mathrm{~L}_{1}$ & $34.33 d-f$ & $32.33 \mathrm{~cd}$ & $26.33 d-f$ & $25.00 \mathrm{c}-\mathrm{e}$ & $36.74 d$ & $34.69 \mathrm{fg}$ & $28.38 \mathrm{e}-\mathrm{g}$ & $26.78 \mathrm{~d}-\mathrm{f}$ & $35.54 \mathrm{e}$ & $33.51 \mathrm{~d}$ & 27.36ef & $25.89 \mathrm{~d}-\mathrm{f}$ \\
\hline $\mathrm{M}_{1} \mathrm{~L}_{2}$ & $33.67 \mathrm{ef}$ & $32.00 \mathrm{~cd}$ & $20.67 \mathrm{hi}$ & $20.00 \mathrm{gh}$ & $36.77 d$ & $34.19 \mathrm{~g}$ & $22.77 \mathrm{i}$ & $20.73 \mathrm{hi}$ & $35.22 \mathrm{e}$ & $33.09 \mathrm{~d}$ & $21.72 \mathrm{~g}$ & 20.36hi \\
\hline $\mathrm{M}_{1} \mathrm{~L}_{3}$ & $34.33 \mathrm{~d}-\mathrm{f}$ & $32.00 \mathrm{~cd}$ & $21.33 \mathrm{~g}-\mathrm{i}$ & $20.67 f-h$ & $36.85 \mathrm{~d}$ & $34.80 \mathrm{fg}$ & $23.89 \mathrm{hi}$ & $21.70 \mathrm{~g}-\mathrm{i}$ & $35.59 \mathrm{e}$ & $33.40 \mathrm{~d}$ & $22.61 \mathrm{~g}$ & $21.19 \mathrm{~g}-\mathrm{i}$ \\
\hline $\mathrm{M}_{1} \mathrm{~L}_{4}$ & $35.00 \mathrm{c}-\mathrm{f}$ & $32.00 \mathrm{~cd}$ & $25.33 \mathrm{~d}-\mathrm{g}$ & $22.33 \mathrm{f}-\mathrm{g}$ & $36.74 d$ & $34.69 \mathrm{fg}$ & $26.30 \mathrm{gh}$ & $24.11 \mathrm{f}-\mathrm{h}$ & $35.87 \mathrm{de}$ & $33.34 d$ & $25.82 \mathrm{f}$ & $23.22 \mathrm{e}-\mathrm{h}$ \\
\hline $\mathrm{M}_{1} \mathrm{~L}_{5}$ & $34.67 d-f$ & $32.33 \mathrm{~cd}$ & $26.00 \mathrm{~d}-\mathrm{f}$ & $23.67 d-g$ & $37.07 \mathrm{~d}$ & $35.02 \mathrm{fg}$ & $27.63 \mathrm{fg}$ & $25.45 \mathrm{e}-\mathrm{g}$ & $35.87 \mathrm{de}$ & $33.68 \mathrm{~d}$ & $26.82 \mathrm{f}$ & $24.56 \mathrm{e}-\mathrm{g}$ \\
\hline $\mathrm{M}_{2} \mathrm{~L}_{1}$ & $36.67 a-d$ & $35.00 \mathrm{ab}$ & $31.33 \mathrm{a}-\mathrm{c}$ & $29.00 \mathrm{ab}$ & $39.25 \mathrm{bc}$ & $36.88 b-f$ & $34.84 \mathrm{bc}$ & $32.65 b c$ & $37.96 \mathrm{bc}$ & $35.94 \mathrm{bc}$ & $33.09 \mathrm{bc}$ & $30.83 b c$ \\
\hline $\mathrm{M}_{2} \mathrm{~L}_{2}$ & $36.67 \mathrm{a}-\mathrm{d}$ & $33.33 \mathrm{bc}$ & $23.67 \mathrm{f}-\mathrm{i}$ & $21.67 \mathrm{e}-\mathrm{g}$ & $38.35 \mathrm{~cd}$ & $35.19 \mathrm{e}-\mathrm{g}$ & $27.27 \mathrm{fg}$ & $24.28 f-h$ & $37.51 \mathrm{c}$ & $34.26 \mathrm{~cd}$ & $25.47 \mathrm{f}$ & $22.98 \mathrm{f}-\mathrm{h}$ \\
\hline $\mathrm{M}_{2} \mathrm{~L}_{3}$ & $36.00 \mathrm{~b}-\mathrm{e}$ & $33.67 \mathrm{bc}$ & $24.33 \mathrm{e}-\mathrm{h}$ & $22.00 \mathrm{e}-\mathrm{g}$ & $38.47 \mathrm{~cd}$ & $36.42 \mathrm{c}-\mathrm{g}$ & $27.95 \mathrm{e}$ & $25.72 \mathrm{e}-\mathrm{g}$ & $37.24 \mathrm{~cd}$ & $35.04 \mathrm{~cd}$ & $26.14 \mathrm{f}$ & $23.86 \mathrm{e}-\mathrm{h}$ \\
\hline $\mathrm{M}_{2} \mathrm{~L}_{4}$ & $38.00 \mathrm{ab}$ & $35.67 \mathrm{a}$ & $28.00 \mathrm{c}-\mathrm{e}$ & $25.33 b-e$ & $39.25 \mathrm{bc}$ & $38.21 \mathrm{a}-\mathrm{d}$ & $31.17 \mathrm{de}$ & $28.98 \mathrm{c}-\mathrm{e}$ & $38.63 a-c$ & $36.94 a b$ & 29.59de & $27.16 \mathrm{c}-\mathrm{e}$ \\
\hline $\mathrm{M}_{2} \mathrm{~L}_{5}$ & $36.00 \mathrm{~b}-\mathrm{e}$ & $33.67 \mathrm{bc}$ & $30.67 a-c$ & $27.00 b-d$ & $38.58 \mathrm{~cd}$ & $36.21 d-g$ & $32.84 \mathrm{bc}$ & $30.65 b-d$ & $37.29 \mathrm{~cd}$ & $34.94 \mathrm{~cd}$ & $31.75 \mathrm{~cd}$ & $28.83 b-d$ \\
\hline $\mathrm{M}_{3} \mathrm{~L}_{1}$ & $39.00 \mathrm{a}$ & $36.00 \mathrm{a}$ & $34.00 \mathrm{a}$ & $32.33 \mathrm{a}$ & 41.08ab & $38.63 \mathrm{a}-\mathrm{c}$ & $38.96 a$ & $36.77 \mathrm{a}$ & $40.04 a$ & $37.32 \mathrm{ab}$ & $36.48 \mathrm{a}$ & $34.55 \mathrm{a}$ \\
\hline $\mathrm{M}_{3} \mathrm{~L}_{2}$ & $37.33 b-c$ & $34.67 \mathrm{ab}$ & $29.33 b-d$ & $23.33 \mathrm{~d}-\mathrm{g}$ & $40.35 a-c$ & $37.46 a-e$ & $30.14 d-f$ & $26.74 d-f$ & $38.84 \mathrm{a}-\mathrm{c}$ & $36.06 a-c$ & $29.74 d \mathrm{de}$ & $25.04 d-g$ \\
\hline $\mathrm{M}_{3} \mathrm{~L}_{3}$ & $38.00 \mathrm{ab}$ & $35.67 \mathrm{a}$ & $31.00 \mathrm{a}-\mathrm{c}$ & $24.33 d-f$ & 41.01ab & $38.96 \mathrm{ab}$ & $31.85 \mathrm{~cd}$ & $28.14 d-f$ & $39.51 \mathrm{ab}$ & $37.32 \mathrm{ab}$ & $31.42 \mathrm{~cd}$ & $26.24 d-f$ \\
\hline $\mathrm{M}_{3} \mathrm{~L}_{4}$ & $38.33 \mathrm{ab}$ & $35.67 \mathrm{a}$ & $32.33 \mathrm{ab}$ & $28.33 b c$ & $41.01 \mathrm{ab}$ & $38.96 \mathrm{ab}$ & $34.96 \mathrm{bc}$ & $32.77 b c$ & $39.67 \mathrm{a}$ & $37.32 \mathrm{ab}$ & $33.65 \mathrm{bc}$ & $30.55 b c$ \\
\hline $\mathrm{M}_{3} \mathrm{~L}_{5}$ & $38.67 \mathrm{a}$ & $36.33 a$ & $33.33 \mathrm{ab}$ & $29.00 \mathrm{ab}$ & $41.68 \mathrm{a}$ & $39.63 a$ & $35.63 \mathrm{~b}$ & $33.44 \mathrm{ab}$ & $40.17 \mathrm{a}$ & $37.98 \mathrm{a}$ & $34.48 \mathrm{ab}$ & $31.22 \mathrm{ab}$ \\
\hline $\mathrm{C}_{1}$ & $33.33 \mathrm{f}$ & $31.00 \mathrm{~d}$ & $20.00 \mathrm{i}$ & $17.67 \mathrm{~h}$ & $33.60 \mathrm{e}$ & $31.48 \mathrm{~h}$ & $21.16 \mathrm{i}$ & $18.99 \mathrm{i}$ & $33.47 \mathrm{f}$ & $31.24 \mathrm{e}$ & $20.58 \mathrm{~g}$ & $18.33 \mathrm{i}$ \\
\hline S.Em. \pm & 0.77 & 0.539 & 1.29 & 1.17 & 0.658 & 0.728 & 1.057 & 1.318 & 0.511 & 0.59 & 0.83 & 1.21 \\
\hline
\end{tabular}

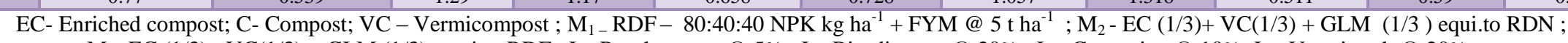

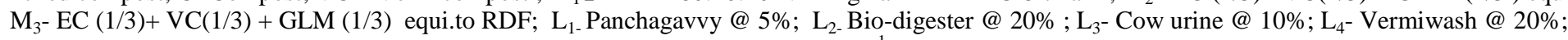
$\mathrm{L}_{5}$ - borax @ 0.2\% + $\mathrm{MgSO}_{4} @ 1 \% ; \mathrm{C}_{1}-\mathrm{RDF}-80: 40: 40 \mathrm{NPK} \mathrm{kg}+\mathrm{FYM} @ 5 \mathrm{tha}^{-1}$ 
Table.4 PSM (cfuX103 / g of soil) population and dehydrogenase activity ( $\mu \mathrm{g}$ TPF /g of soil / day) observed in cotton soil as influenced by organic manures, GLM, liquid organic manures and micronutrients

\begin{tabular}{|c|c|c|c|c|c|c|c|c|c|c|c|c|}
\hline \multirow[t]{2}{*}{ Treatment } & \multicolumn{4}{|c|}{2010} & \multicolumn{4}{|c|}{2011} & \multicolumn{4}{|c|}{ Pooled } \\
\hline & $\begin{array}{c}\text { PSM } \\
\text { at } 60 \text { DAS }\end{array}$ & PSM at 90DAS & $\begin{array}{l}\text { Dehydrogenase } \\
\text { at } 60 \text { DAS }\end{array}$ & $\begin{array}{c}\text { Dehydrogen } \\
\text { ase } \\
\text { at } 90 \text { DAS }\end{array}$ & $\begin{array}{l}\text { PSM at } \\
\text { 60 DAS }\end{array}$ & $\begin{array}{c}\text { PSM at } 90 \\
\text { DAS }\end{array}$ & $\begin{array}{l}\text { Dehydrogenase } \\
\text { at } 60 \text { DAS }\end{array}$ & $\begin{array}{l}\text { Dehydrogenase } \\
\text { at } 90 \text { DAS }\end{array}$ & $\begin{array}{l}\text { PSM at } \\
\text { 60 DAS }\end{array}$ & $\begin{array}{c}\text { PSM at } 90 \\
\text { DAS }\end{array}$ & $\begin{array}{l}\text { Dehydrogenase } \\
\text { at } 60 \text { DAS }\end{array}$ & $\begin{array}{l}\text { Dehydrogenase } \\
\text { at } 90 \text { DAS }\end{array}$ \\
\hline \multicolumn{13}{|c|}{ Organic Manure (M) } \\
\hline $\mathrm{M}_{1}$ & $16.73 \mathrm{c}$ & $14.82 \mathrm{c}$ & $8.51 \mathrm{c}$ & $7.09 \mathrm{~b}$ & $17.77 \mathrm{c}$ & $15.81 \mathrm{c}$ & $10.14 \mathrm{c}$ & $8.42 \mathrm{c}$ & $17.25 \mathrm{c}$ & $15.31 \mathrm{c}$ & $9.33 \mathrm{c}$ & $7.76 \mathrm{c}$ \\
\hline $\mathrm{M}_{2}$ & $20.80 \mathrm{~b}$ & $18.47 \mathrm{~b}$ & $9.48 \mathrm{~b}$ & $8.02 \mathrm{a}$ & $23.20 \mathrm{~b}$ & $21.42 \mathrm{~b}$ & $12.54 \mathrm{~b}$ & $10.75 b$ & $22.00 \mathrm{~b}$ & $19.94 \mathrm{~b}$ & $11.01 \mathrm{~b}$ & $9.38 b$ \\
\hline $\mathrm{M}_{3}$ & $24.67 \mathrm{a}$ & $22.73 a$ & $9.85 \mathrm{a}$ & $8.22 \mathrm{a}$ & $27.64 \mathrm{a}$ & $25.89 \mathrm{a}$ & $14.13 \mathrm{a}$ & $12.32 \mathrm{a}$ & $26.15 \mathrm{a}$ & $24.31 \mathrm{a}$ & $11.99 \mathrm{a}$ & $10.27 \mathrm{a}$ \\
\hline S.Em. \pm & 0.478 & 0.499 & 0.0935 & 0.129 & 0.202 & 0.271 & 0.149 & 0.171 & 0.245 & 0.421 & 0.029 & 0.072 \\
\hline \multicolumn{13}{|c|}{ Foliar spray of liquid manures + micronutrients (L) } \\
\hline $\mathrm{L}_{1}$ & $22.89 \mathrm{a}$ & $20.56 \mathrm{a}$ & $9.69 \mathrm{a}$ & $8.27 \mathrm{a}$ & $25.30 \mathrm{a}$ & $23.26 \mathrm{a}$ & $12.71 \mathrm{a}$ & $10.97 \mathrm{a}$ & $24.09 \mathrm{a}$ & $21.91 \mathrm{a}$ & $11.20 \mathrm{a}$ & $9.62 \mathrm{a}$ \\
\hline $\mathrm{L}_{2}$ & $18.89 \mathrm{~d}$ & $17.78 \mathrm{~cd}$ & $8.87 \mathrm{~b}$ & $7.04 \mathrm{~b}$ & $20.20 \mathrm{c}$ & $19.22 \mathrm{c}$ & $11.68 \mathrm{c}$ & $9.84 \mathrm{c}$ & $19.55 \mathrm{~d}$ & $18.50 \mathrm{~cd}$ & $10.27 \mathrm{~b}$ & $8.44 \mathrm{c}$ \\
\hline $\mathrm{L}_{3}$ & $19.33 \mathrm{~cd}$ & $16.89 \mathrm{~d}$ & $8.99 b$ & 7.57ab & $21.58 \mathrm{c}$ & $19.53 \mathrm{c}$ & $12.03 \mathrm{bc}$ & $10.27 \mathrm{bc}$ & $20.46 c$ & $18.21 \mathrm{ab}$ & $10.51 \mathrm{~b}$ & $8.92 b$ \\
\hline $\mathrm{L}_{4}$ & $20.89 \mathrm{bc}$ & $18.67 \mathrm{bc}$ & $9.35 \mathrm{a}$ & $7.92 \mathrm{a}$ & $23.19 \mathrm{~b}$ & $21.14 \mathrm{~b}$ & $12.38 \mathrm{ab}$ & $10.62 \mathrm{ab}$ & $22.04 \mathrm{~b}$ & $19.91 \mathrm{ab}$ & $10.86 \mathrm{a}$ & $9.27 \mathrm{ab}$ \\
\hline $\mathrm{L}_{5}$ & $21.67 \mathrm{ab}$ & $19.47 \mathrm{ab}$ & $9.51 \mathrm{a}$ & $8.09 a$ & $24.08 \mathrm{ab}$ & $22.03 a b$ & $12.56 \mathrm{ab}$ & 10.79ab & $22.87 \mathrm{~b}$ & $20.75 \mathrm{ab}$ & $11.03 \mathrm{a}$ & $9.44 a$ \\
\hline S.Em. \pm & 0.482 & 0.497 & 0.119 & 0.245 & 0.521 & 0.542 & 0.189 & 0.202 & 0.289 & 0.413 & 0.111 & 0.159 \\
\hline \multicolumn{13}{|c|}{ Interactions (MXL) } \\
\hline $\mathrm{M}_{1} \mathrm{~L}_{1}$ & $18.67 \mathrm{~d}-\mathrm{g}$ & $16.33 \mathrm{ef}$ & $8.89 \mathrm{e}-\mathrm{g}$ & $7.47 \mathrm{~b}-\mathrm{g}$ & 19.93f-h & $17.88 \mathrm{ef}$ & $10.59 \mathrm{f}$ & $8.83 \mathrm{f}$ & 19.30gh & $17.11 \mathrm{~g}-\mathrm{i}$ & $9.74 \mathrm{f}$ & $8.15 f g$ \\
\hline $\mathrm{M}_{1} \mathrm{~L}_{2}$ & $15.33 \mathrm{~g}$ & $13.33 \mathrm{~g}$ & $8.17 \mathrm{~h}$ & $6.77 \mathrm{~g}$ & $16.12 \mathrm{i}$ & $14.48 \mathrm{gh}$ & $9.50 \mathrm{gh}$ & $7.96 \mathrm{fg}$ & $15.73 \mathrm{j}$ & $13.91 \mathrm{jk}$ & $8.84 \mathrm{~h}$ & $7.37 \mathrm{gh}$ \\
\hline $\mathrm{M}_{1} \mathrm{~L}_{3}$ & $15.67 \mathrm{~g}$ & $14.33 \mathrm{fg}$ & $8.22 \mathrm{~h}$ & $6.80 \mathrm{fg}$ & $16.62 \mathrm{i}$ & $14.57 \mathrm{gh}$ & $9.92 \mathrm{f}-\mathrm{h}$ & $8.16 \mathrm{f}$ & $16.14 \mathrm{j}$ & $14.45 \mathrm{jk}$ & $9.07 \mathrm{gh}$ & $7.48 \mathrm{gh}$ \\
\hline $\mathrm{M}_{1} \mathrm{~L}_{4}$ & $16.67 \mathrm{fg}$ & $14.67 \mathrm{fg}$ & $8.56 \mathrm{gh}$ & 7.13d-g & 17.59hi & $15.55 \mathrm{f}-\mathrm{h}$ & $10.25 f-g$ & $8.50 \mathrm{f}$ & $17.13 \mathrm{ij}$ & $15.11 \mathrm{i}-\mathrm{k}$ & $9.40 \mathrm{fg}$ & $7.82 \mathrm{~g}$ \\
\hline $\mathrm{M}_{1} \mathrm{~L}_{5}$ & $17.33 \mathrm{fg}$ & $15.42 \mathrm{fg}$ & $8.72 \mathrm{f}-\mathrm{h}$ & $7.30 \mathrm{c}-\mathrm{g}$ & $18.59 \mathrm{~g}-\mathrm{i}$ & $16.55 \mathrm{fg}$ & $10.44 f-g$ & $8.66 \mathrm{f}$ & 17.96hi & $15.98 \mathrm{~h}-\mathrm{j}$ & $9.58 \mathrm{fg}$ & $7.98 \mathrm{fg}$ \\
\hline $\mathrm{M}_{2} \mathrm{~L}_{1}$ & $22.33 \mathrm{e}-\mathrm{g}$ & $20.00 \mathrm{~cd}$ & 9.86a-d & $8.43 a-d$ & $24.97 \mathrm{~cd}$ & $22.93 \mathrm{~cd}$ & $12.97 \mathrm{~b}-\mathrm{d}$ & $11.21 \mathrm{~cd}$ & $23.65 \mathrm{~cd}$ & $21.47 \mathrm{de}$ & $11.42 \mathrm{c}$ & $9.82 \mathrm{~b}-\mathrm{d}$ \\
\hline $\mathrm{M}_{2} \mathrm{~L}_{2}$ & $19.33 c-f$ & $18.33 \mathrm{de}$ & $9.05 \mathrm{e}-\mathrm{g}$ & $7.43 \mathrm{~b}-\mathrm{g}$ & $20.94 \mathrm{e}-\mathrm{g}$ & $20.22 \mathrm{de}$ & $11.88 \mathrm{e}$ & $9.98 \mathrm{e}$ & $20.14 f g$ & $19.28 \mathrm{e}-\mathrm{g}$ & $10.47 \mathrm{e}$ & $8.71 \mathrm{ef}$ \\
\hline $\mathrm{M}_{2} \mathrm{~L}_{3}$ & $20.00 \mathrm{c}-\mathrm{e}$ & $16.33 \mathrm{ef}$ & $9.22 \mathrm{~d}-\mathrm{f}$ & $7.80 \mathrm{a}-\mathrm{g}$ & $22.45 \mathrm{~d}-\mathrm{f}$ & 20.41de & $12.33 \mathrm{de}$ & $10.57 \mathrm{de}$ & $21.23 \mathrm{ef}$ & $18.37 \mathrm{f}-\mathrm{h}$ & $10.78 \mathrm{de}$ & $9.19 \mathrm{de}$ \\
\hline $\mathrm{M}_{2} \mathrm{~L}_{4}$ & $21.00 \mathrm{~cd}$ & $18.67 \mathrm{de}$ & 9.56b-e & $8.13 a-e$ & $23.64 c-e$ & $21.60 \mathrm{~cd}$ & $12.67 \mathrm{c}-\mathrm{e}$ & 10.91de & $22.32 \mathrm{de}$ & $20.13 d-f$ & $11.11 \mathrm{~cd}$ & $9.52 \mathrm{c}-\mathrm{e}$ \\
\hline $\mathrm{M}_{2} \mathrm{~L}_{5}$ & $21.33 \mathrm{~cd}$ & $19.00 \mathrm{c}-\mathrm{e}$ & $9.72 a-d$ & $8.30 \mathrm{a}-\mathrm{d}$ & $23.97 \mathrm{~cd}$ & $21.93 \mathrm{~cd}$ & $12.83 \mathrm{~b}-\mathrm{e}$ & $11.07 \mathrm{c}-\mathrm{e}$ & $22.65 c-e$ & $20.47 d-f$ & $11.28 \mathrm{~cd}$ & $9.69 b-d$ \\
\hline $\mathrm{M}_{3} \mathrm{~L}_{1}$ & $27.67 \mathrm{a}$ & $25.33 \mathrm{a}$ & $10.33 a$ & $8.90 \mathrm{a}$ & $31.00 \mathrm{a}$ & $28.95 \mathrm{a}$ & $14.56 \mathrm{a}$ & $12.86 \mathrm{a}$ & $29.33 a$ & $27.14 \mathrm{a}$ & $12.45 \mathrm{a}$ & $10.88 \mathrm{a}$ \\
\hline $\mathrm{M}_{3} \mathrm{~L}_{2}$ & 22 & $21.67 \mathrm{bc}$ & $9.38 \mathrm{c}-\mathrm{f}$ & $6.93 \mathrm{e}-\mathrm{g}$ & $23.54 \mathrm{c}-\mathrm{e}$ & $22.95 \mathrm{~cd}$ & $13.64 \mathrm{a}-\mathrm{c}$ & $11.57 b-d$ & $22.77 c-e$ & $22.31 \mathrm{~cd}$ & $11.51 \mathrm{c}$ & $9.25 c-e$ \\
\hline $\mathrm{M}_{3} \mathrm{~L}_{3}$ & $22.33 b c$ & $20.00 \mathrm{~cd}$ & $9.52 \mathrm{~b}-\mathrm{e}$ & $8.10 \mathrm{a}-\mathrm{f}$ & $25.66 c$ & $23.62 \mathrm{c}$ & $13.83 \mathrm{ab}$ & $12.06 \mathrm{a}-\mathrm{c}$ & $24.00 \mathrm{c}$ & $21.81 \mathrm{de}$ & $11.68 \mathrm{bc}$ & $10.08 \mathrm{a}-\mathrm{c}$ \\
\hline $\mathrm{M}_{3} \mathrm{~L}_{4}$ & $25.00 \mathrm{ab}$ & $22.67 \mathrm{~b}$ & $9.92 \mathrm{a}-\mathrm{c}$ & $8.50 \mathrm{a}-\mathrm{c}$ & $28.33 b$ & $26.29 b$ & $14.22 \mathrm{a}$ & $12.46 \mathrm{ab}$ & $26.67 b$ & $24.48 b c$ & $12.07 \mathrm{ab}$ & $10.48 \mathrm{ab}$ \\
\hline $\mathrm{M}_{3} \mathrm{~L}_{5}$ & $26.33 a$ & $24.00 \mathrm{ab}$ & $10.09 \mathrm{ab}$ & $8.67 \mathrm{ab}$ & 29.66ab & 27.62ab & $14.39 \mathrm{a}$ & 12.63ab & $28.00 \mathrm{ab}$ & $25.81 \mathrm{ab}$ & $12.24 \mathrm{a}$ & $10.65 \mathrm{a}$ \\
\hline $\mathrm{C}_{1}$ & $16.00 \mathrm{~g}$ & $12.67 \mathrm{~g}$ & $8.20 \mathrm{~h}$ & $6.77 \mathrm{~g}$ & $16.67 \mathrm{i}$ & $13.48 \mathrm{~h}$ & $9.07 \mathrm{~h}$ & $6.96 \mathrm{~g}$ & $16.34 \mathrm{j}$ & $13.08 \mathrm{k}$ & $8.64 \mathrm{~h}$ & $6.87 \mathrm{~h}$ \\
\hline S.Em. \pm & 0.986 & 0.878 & 0.207 & 0.395 & 0.874 & 0.876 & 0.321 & 0.351 & 0.515 & 0.817 & 0.179 & 0.261 \\
\hline
\end{tabular}

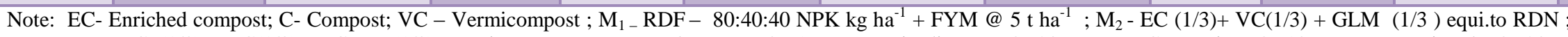
$\mathrm{M}_{3^{-}}$EC (1/3)+ VC(1/3) + GLM (1/3) equi.to RDF; $\mathrm{L}_{1}$. Panchagavvy @ 5\%; $\mathrm{L}_{2}$ Bio-digester @ 20\%; $\mathrm{L}_{3^{-}}$Cow urine@10\%; $\mathrm{L}_{4^{-}}$Vermiwash @20\%; $\mathrm{L}_{5}$ - borax @ $0.2 \%+\mathrm{MgSO}_{4} @ 1 \% ; \mathrm{C}_{1}-\mathrm{RDF}-80: 40: 40 \mathrm{NPK} \mathrm{kg}+\mathrm{FYM} @ 5 \mathrm{tha}^{-1}$ 
Table.5 Phosphatase activity ( $\mu \mathrm{g}$ pnpTPF /g of soil / hr) and $\mathrm{CO}_{2}$ exchange rate $\left(\mathrm{mg}\right.$ of $\mathrm{CO}_{2} / \mathrm{hr} / 100 \mathrm{~g}$ soil) in cotton soil as influenced by organic manures, GLM, liquid organic manures and micronutrients

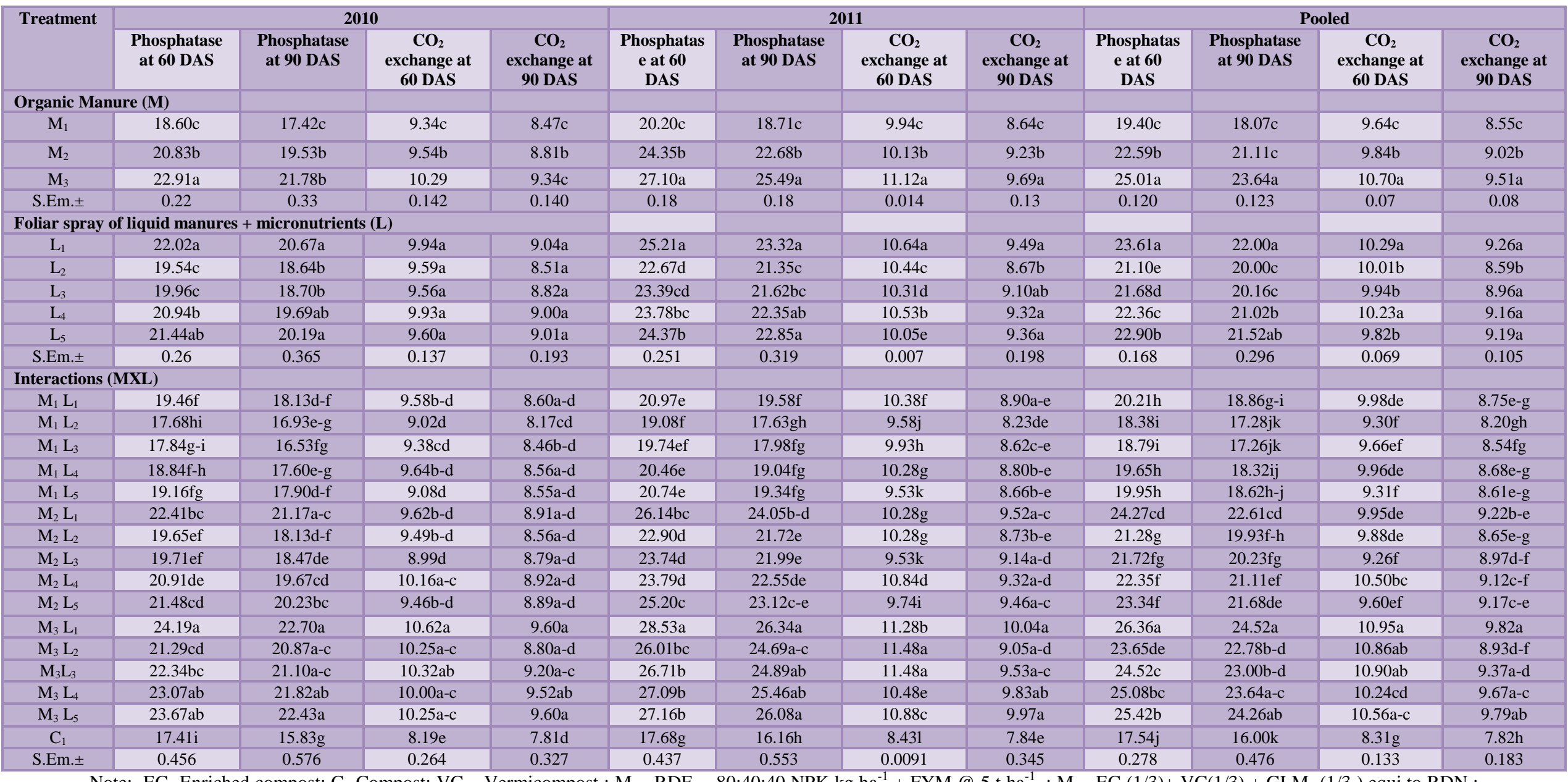

Note: EC- Enriched compost; C- Compost; VC - Vermicompost ; $\mathrm{M}_{1-} \mathrm{RDF}-80: 40: 40 \mathrm{NPK} \mathrm{kg} \mathrm{ha}{ }^{-1}+\mathrm{FYM} @ 5 \mathrm{t} \mathrm{ha}{ }^{-1} ; \mathrm{M}_{2}-\mathrm{EC}(1 / 3)+\mathrm{VC}(1 / 3)+\mathrm{GLM}(1 / 3)$ equi.to RDN ; $\mathrm{M}_{3^{-}}$EC $(1 / 3)+\mathrm{VC}(1 / 3)+\mathrm{GLM}(1 / 3)$ equi.to RDF; L $\mathrm{L}_{1}$. Panchagavvy @ 5\%; L $\mathrm{L}_{2}$ Bio-digester @ 20\%; $\mathrm{L}_{3^{-}}$Cow urine@10\%; $\mathrm{L}_{4}$ - Vermiwash @ 20\%; $\mathrm{L}_{5}$ - borax @ 0.2\%+ $\mathrm{MgSO}_{4} @ 1 \% ; \mathrm{C}_{1}-\mathrm{RDF}-80: 40: 40 \mathrm{NPK} \mathrm{kg}+\mathrm{FYM} @ 5 \mathrm{tha}^{-1}$ 
It was higher by $3.42,9.72$ and 4.38 per cent over FYM @ $5 \mathrm{tha}^{-1}+$ RDF. Organic manures in conjunction with foliar spray of panchagavya recorded significantly higher available soil $\mathrm{N}, \mathrm{P}_{2} \mathrm{O}_{5}$ and $\mathrm{K}_{2} \mathrm{O}(279.46,28.38$ and $324.49 \mathrm{~kg} \mathrm{ha}^{-1}$, respectively) as compared to foliar spray of bio-digester @ 20\% and was on par with borax @0.2\%+. $\mathrm{MgSO}_{4} @ 1 \%$ and vermiwash@20\% and cow urine@10 $\%$.

Among the different treatment combinations, application of EC (1/3) + VC (1/3) + gliricidia GLM (1/3) equivalent to RDF with foliar spray of panchagavya @ 5\% recorded significantly higher available soil $\mathrm{N}, \mathrm{P}_{2} \mathrm{O}_{5}$ and $\mathrm{K}_{2} \mathrm{O}$ (284.4, 29.7 and $330.0 \mathrm{~kg} \mathrm{ha}^{-1}$, respectively) accounting for 5.66, 23.51 and 13.40 per cent, higher available NPK over RDF + FYM (269.2, 24.1 and $\left.291.0 \mathrm{~kg} \mathrm{ha}^{-1}\right)$ and was on par with EC (1/3) + VC $(1 / 3)+$ gliricidia GLM (1/3) equivalent to RDF with borax@0.2\%+. $\mathrm{MgSO}_{4} @ 1 \%, \mathrm{EC}(1 / 3)+$ VC $(1 / 3)+$ gliricidia GLM (1/3) equivalent to RDF with vermiwash @ 20\% and EC (1/3) + VC $(1 / 3)+$ gliricidia GLM (1/3) equivalent to RDF with cow urine@10\%.

Application of EC (1/3) + VC (1/3) + GLM (1/3) equivalent to RDF with foliar spray of panchagavya@5\% recorded higher gain in available $\mathrm{N}, \mathrm{P}_{2} \mathrm{O}_{5}$ and $\mathrm{K}_{2} \mathrm{O}$ over $\mathrm{RDF}+\mathrm{FYM}$ and closely followed by EC (1/3) + VC (1/3) + gliricidia GLM (1/3) equivalent to RDF with borax@0.2\%+. $\mathrm{MgSO}_{4} @ 1 \%$. Higher soil available $\mathrm{N}, \mathrm{P}_{2} \mathrm{O}_{5}$ and $\mathrm{K}_{2} \mathrm{O}$ may be due to higher soil microbial in soil activity. These results are in confirmity with findings of Kademani et al., (2004). Integrated application of EC (1/3) + VC (1/3) + gliricidia GLM (1/3) equivalent to RDF recorded significantly higher actinomycetes, phosphorus solubilising bacteria, $\mathrm{N}_{2}$-fixers, enzymes mainly phosphatase and dehydrogenase activity and soil respiration rate $\left(73.19 \mathrm{cfu} \times 10^{6} \% \mathrm{~g}\right.$ of soil, $26.84 \mathrm{cfu}$
$\mathrm{X} 10^{3} / \mathrm{g}$ of soil, $39.65 \mathrm{cfu} \times 10^{2} / \mathrm{g}$ of soil

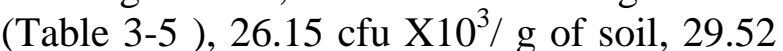
cfu $X 10^{3} / \mathrm{g}$ of soil, $25.01 \mu \mathrm{pnp} / \mathrm{g}$ of soil/hr, $11.99 \mu \mathrm{TPF} / \mathrm{g}$ of soil/day, and $9.51 \mathrm{mg}$ of $\mathrm{C}$ or $\mathrm{CO}_{2 /} \mathrm{hr} / 100 \mathrm{~g}$ of soil respectively) at 60 DAS as compared to application of FYM @ 5 $\mathrm{t} \mathrm{ha}^{-1}+\mathrm{RDF}$. The foliar spray of panchagavya @ 5\% in combination with organic manures recorded significantly higher population of actinomycetes, $\mathrm{N}_{2}$-fixers and PSM, phosphatase and dehydrogenase enzyme activity and soil respiration rate over foliar spray of bio-digester @ 20\% with organic manures and was on parwith borax@0.2\%+ $\mathrm{MgSO}_{4} @ 1 \%$ and vermiwash@20\%.

Among the different treatment combinations, application of EC (1/3) + VC (1/3) + gliricidia GLM (1/3) equivalent to RDF with foliar spray of panchagavya @ 5\% recorded significantly higher actinomycetes, $\mathrm{N}_{2}$-fixers and P-solubilizer, phosphatase and dehydrogenase enzyme activity and soil respiration rate over RDF + FYM and was on par with EC $(1 / 3)+\mathrm{VC}(1 / 3)+$ gliricidia GLM (1/3) equivalent to RDF with borax @ $0.2 \%+\mathrm{MgSO}_{4} @ 1 \%, \mathrm{EC}(1 / 3)+\mathrm{VC}(1 / 3)+$ gliricidia GLM (1/3) equivalent to RDF with vermiwash@ 20\% and EC (1/3) + VC (1/3) + gliricidia GLM (1/3) equivalent to RDF with cow urine @ 10\%. These results are in line with the findings of Solaiappan (2004). Finally concluded that combined application of EC (1/3) + VC (1/3) + gliricidia GLM (1/3) equivalent to RDN with foliar spray of panchagavya@5\% improved soil properties.

\section{References}

Anonymous, 2011, Area, production and yield of cotton in India (major states). Technical Report, Directorate of Economics and Statistics, Department of Agriculture and Cooperation, New Delhi, p. 77. 
Lokesh, B. S., Malabasari., B.S.,Vyakarnal, N. K., Biradarpatil, N.K. and Kotikal., 2008, Studies on physico-chemical properties of cotton growing soil. $J$. Cotton Res. Dev. 23(1): 60 - 63.

Sanjutha, S., Subramanian, C., Indu Rani and Maheswari, J., 2008, Integrated Nutrient Management in Andrographis paniculata. Res. J. Agric. Biol. Sci., 4 (2): 141-145.

Tolanur, S. I., 2008, Integrated effect of organic manuring and inorganic fertilizer on yield and uptake of micronutrients by chickpea in Vertisol. Legume Res., 31 (3): 184-187.
Boomathi, N., Suganya Kanna, S. and Jeyarani, S., 2005, "Panchagavya" - A gift from our mother's nature. Agrobios. News lett., 4 (3): 20-21.

Kademani, M. B., Radder, B. M. and Hebsur, N. S., 2004, Effect of organic and inorganic fertilizers on availability and uptake of nutrients by sunflower in vertisols of Malaprabha command. Karnataka J. Agri. Sci., 16 (1): 48-53.

Solaiappan, U., 2002, Effect of inorganic fertilizer and organic manure on cottonsorghum rotation in rainfed Vertisols. Madras Agric. J., 89 (7-9): 448-450.

\section{How to cite this article:}

Rudragouda F. Channagouda. 2019. Microbial Population and Soil Health under Organic Cotton Production System. Int.J.Curr.Microbiol.App.Sci. 8(03): 2519-2528.

doi: https://doi.org/10.20546/ijcmas.2019.803.299 